\title{
Experimental method of detecting relic neutrino by atomic de-excitation
}

\author{
M. Yoshimura, N. Sasao ${ }^{\dagger}$, and M. Tanaka \\ Center of Quantum Universe, Faculty of Science, Okayama University \\ Tsushima-naka 3-1-1 Kita-ku Okayama 700-8530 Japan \\ $\dagger$ Research Core for Extreme Quantum World, Okayama University \\ Tsushima-naka 3-1-1 Kita-ku Okayama 700-8530 Japan \\ $\ddagger$ Department of Physics, Graduate School of Science, Osaka University, Toyonaka, Osaka 560-0043, Japan
}

\begin{abstract}
The cosmic background neutrino of temperature $1.9 \mathrm{~K}$ affects rates of radiative emission of neutrino pair (RENP) from metastable excited atoms, since its presence blocks the pair emission by the Pauli exclusion principle. We quantitatively investigate how the Pauli blocking distorts the photon energy spectrum and calculate its sensitivity to cosmic parameters such as the neutrino temperature and its chemical potential. Important quantities for high sensitivities to these parameter measurement are found to be the level spacing of atomic de-excitation and the unknown mass value of lightest neutrino, in particular their mutual relation.
\end{abstract}

PACS numbers $\quad 13.15 .+\mathrm{g}, 14.60 . \mathrm{Pq}, 98.80 . \mathrm{Es}$

Keywords relic neutrino, Pauli exclusion principle, big bang cosmology, nucleo-synthesis, neutrino mass, cosmological lepton asymmetry 
The relic cosmic neutrino of temperature $(4 / 11)^{1 / 3} T_{\gamma} \sim 1.9 \mathrm{~K}$ (with $T_{\gamma}$ the cosmic microwave temperature) is undoubtedly one of the most important predictions of the big bang cosmology 2. Detection of relic neutrino would give a strong support for nucleo-synthesis theory that explains the origin of cosmic light elements such as ${ }^{4} \mathrm{He}$. Various ideas of experimental method of relic neutrino detection have been discussed in the literature 3, 4, 5, 6, 7, 8, 9, 10, 11, 12.

In the present work we propose a new experimental method using excited atomic targets. The idea is based on the fact that radiative emission of neutrino pair (RENP) [13 is affected by the Pauli blocking of ambient cosmic neutrinos [14. We shall give an answer to the fundamental issue of how sensitive the Pauli blocking effect is to determination of cosmological parameters, the neutrino temperature and the chemical potential which is related to the lepton asymmetry of our universe.

The process we use is atomic de-excitation from a metastable state $|e\rangle ;|e\rangle \rightarrow|g\rangle+\gamma+\nu_{i} \bar{\nu}_{j}$ (anti-neutrino $\bar{\nu}_{j}$ is identical to $\nu_{j}$ in the case of Majorana neutrino). Energy spectrum of the photon $\gamma$ and parity violating quantities such as the asymmetry of rates under the magnetic field reversal [15] are measured in RENP. $\nu_{i}(i=1,2,3)$ is a mass eigenstate of neutrinos and a mixture of neutrino species $\nu_{e}, \nu_{\mu}, \nu_{\tau}$ that appear in the weak decay of elementary particles. Neutrino oscillation experiments [16 have determined two mass squared differences, $(\sim 50 \mathrm{meV})^{2}$ and $(\sim 10 \mathrm{meV})^{2}$, and three mixing angles in a theoretical framework of an extended standard gauge theory where finite neutrino masses and $3 \times 3$ unitary mixing are introduced as an extra assumption. RENP process predicts a continuous photon energy spectrum at $\omega<\omega_{i j}$ (we use the natural unit of $\hbar=c=k_{B}=1$ such that $\omega$ is the photon energy). Six thresholds are given by $\omega_{i j}=\epsilon_{e g} / 2-\left(m_{i}+m_{j}\right)^{2} /\left(2 \epsilon_{e g}\right)$ with $\epsilon_{e g}$ the level spacing of excitation. Since RENP occurs via stimulated photon emission by trigger lasers, decomposition into neutrino mass eigen-states is made possible by the excellent resolution of trigger laser frequencies. RENP experimental project [13] has been proposed to determine the smallest neutrino mass $m_{0}$, to distinguish the Majorana neutrino from the Dirac neutrino and to determine remaining elements of the mixing matrix, $\mathrm{CP}$ violating $(\mathrm{CPV})$ phases including the ones intrinsic to the Majorana neutrino.

Under the ambient relic neutrino background RENP rates are reduced by the product of Pauli blocking factors $\left(1-f_{i}\right)\left(1-\bar{f}_{j}\right)$ where $f_{i}, \bar{f}_{j}$ are the momentum distribution functions for mass eigen-states $\nu_{i}, \bar{\nu}_{j}$. The Einstein relation in the expanding universe is $E=\sqrt{p^{2}+m^{2} /(z+1)^{2}}, z+1=a(t) / a\left(t_{d}\right)$ where $a(t), t_{d}, z$ are the cosmic scale factor at the present time, the decoupling time and the red shift factor since the neutrino decoupling. To a good approximation (ignoring the momentum region, $p<O(100 \mathrm{meV}) /(z+1) \sim O\left(10^{-11}\right)$ eV of extremely small phase space), the neutrino mass term can be neglected in the distribution functions even at the present epoch. The distribution function after the neutrino decoupling changes under the gravity of the expanding universe and its present form is given by $f_{i}(p)=1 /\left(e^{p / T_{\nu}-\mu_{d} / T_{d}}+1\right)$ where $T_{\nu}$ is the effective neutrino temperature at present given by $(4 / 11)^{1 / 3} T_{\gamma} \sim 1.9 \mathrm{~K}$. The quantity related to the chemical potential, $\mu_{d} / T_{d}$, is the ratio of the chemical potential to the temperature at the epoch of neutrino decoupling [17. For $\bar{\nu}_{j}$ the chemical potential is sign reversed; $\mu_{d} \rightarrow-\mu_{d}$. The upper bound allowed by nucleo-synthesis is of $O(1)$ [18. Some cosmological models predict a large $\mu_{d} / T_{d}$ [19].

The underlying assumption for description in terms of a single neutrino temperature $T_{\nu}$ is that no dramatic entropy generation occurs at the epoch between decoupling of $\nu_{\mu}, \nu_{\tau}$ and electron neutrino $\nu_{e}$, since their decoupling temperatures are close; $\sim 1.9 \mathrm{MeV}$ for $\nu_{e}$ decoupling, and $\sim 3.1 \mathrm{MeV}$ for $\nu_{\mu}, \nu_{\tau}$ decoupling [20. The measurement of $1.9 \mathrm{~K}$ neutrino temperature different from the microwave temperature $2.7 \mathrm{~K}$ is a clear indication of physical process that occurred at earlier epochs of a few seconds after the big bang; electron-positron annihilation.

Effect of the gravitational clustering is expected to be small in the neutrino mass range of $<O(100) \mathrm{meV}$ considered below. The gravitational clustering of massive neutrinos enhances distortion of the spectrum further than the case without the clustering, thus gives a brighter prospect of relic neutrino detection. A simple rough estimate of the clustering effect is to multiply the ratio of the number density of relevant neutrino in our galaxy to the cosmic density $3 \zeta(3) T_{\nu}^{3} /\left(2 \pi^{2}\right) \sim 110 \mathrm{~cm}^{-3}$. This ratio may be calculated, if necessary, by solving the gravitational collapse of massive, but non-interacting particles under the gravity of cold dark matter [21], [22].

Spectrum shape functions previously derived without the Pauli blocking effect [23, 24] are modified by $(1-$ $\left.f_{i}\right)\left(1-\bar{f}_{j}\right)$ for pair production of $\nu_{i} \bar{\nu}_{j}$ at $\omega<\omega_{i j}$. The spectral shape function $F^{A}(A=M$ for the nuclear monopole contribution of three thresholds $\omega_{i i}[23$ and $A=S$ for the electron spin contribution of much smaller absolute rates [24], two cases being applicable to atoms of different quantum numbers) for the neutrino pair production of masses $m_{i}, m_{j}$ is calculated as an integral over one of the neutrino energies;

$$
\begin{gathered}
F_{i j}^{A}\left(\omega ; T_{\nu}\right)=\frac{1}{8 \pi \omega} \int_{E_{-}}^{E_{+}} d E_{1} g_{i j}^{A}\left(E_{1}\right) \cdot\left(1-f\left(\sqrt{E_{1}^{2}-m_{i}^{2}}\right)\right)\left(1-\bar{f}\left(\sqrt{\left(\epsilon_{e g}-\omega-E_{1}\right)^{2}-m_{j}^{2}}\right)\right), \\
g_{i i}^{M}(E)=-E^{2}+\left(\epsilon_{e g}-\omega\right) E+\frac{1}{2} m_{i}^{2}-\frac{1}{4} \epsilon_{e g}\left(\epsilon_{e g}-2 \omega\right)+\delta_{M} \frac{m_{i}^{2}}{2} \\
g_{i j}^{S}(E)=-\frac{1}{3} E^{2}+\frac{1}{3}\left(\epsilon_{e g}-\omega\right) E+\frac{1}{12} \epsilon_{e g}\left(\epsilon_{e g}-2 \omega\right)-\frac{1}{12}\left(m_{i}^{2}+m_{j}^{2}\right)-\delta_{M} \frac{m_{i} m_{j}}{2}, \\
E_{ \pm}=\frac{1}{2}\left(\left(\epsilon_{e g}-\omega\right)\left(1+\frac{m_{i}^{2}-m_{j}^{2}}{\epsilon_{e g}\left(\epsilon_{e g}-2 \omega\right)}\right) \pm \omega \Delta_{i j}(\omega)\right), \quad \Delta_{i j}(\omega)=\left\{\left(1-\frac{\left(m_{i}+m_{j}\right)^{2}}{\epsilon_{e g}\left(\epsilon_{e g}-2 \omega\right)}\right)\left(1-\frac{\left(m_{i}-m_{j}\right)^{2}}{\epsilon_{e g}\left(\epsilon_{e g}-2 \omega\right)}\right)\right\}^{1 / 2} .
\end{gathered}
$$




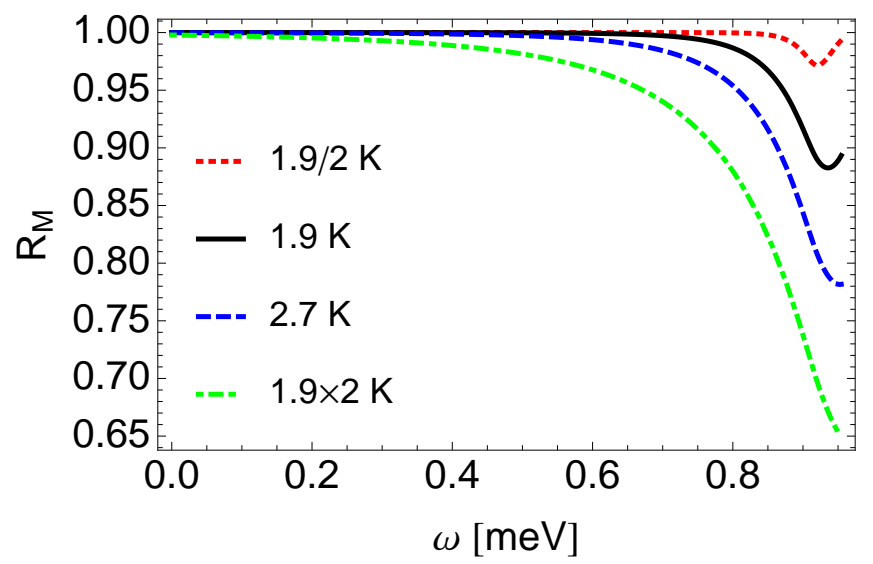

Figure 1: Spectral distortion $R_{M}(\omega)$ caused by the Pauli blocking of relic neutrinos, $T_{\nu}=1.9 / 2 \mathrm{~K}$ in dotted red, 1.9 $\mathrm{K}$ in solid black, $2.7 \mathrm{~K}$ in dashed blue and $1.9 \times 2 \mathrm{~K}$ in dash-dotted green, all assuming $m_{0}=5 \mathrm{meV}, \epsilon_{e g}=11 \mathrm{meV}$ and the zero chemical potential. Distortions are identical for the two cases of NH and IH.

times factors related to atomic matrix elements and energy denominators in perturbation theory [13. These atomic factors cancel out in the ratio of rates, the rate with to the rate without the Pauli blocking. Eq.(1) is a function of photon energy $\omega$, depending on five parameters, two cosmological ones $T_{\nu}, \mu_{d} / T_{d}$, two neutrino masses, $m_{i}, m_{j}$, and the atomic level spacing $\epsilon_{e g} . \delta_{M}=1$ for Majorana neutrinos, arising from the interference term of identical fermions, and $\delta_{M}=0$ in its absence for Dirac neutrinos. In the numerical calculations below we present results for the Majorana case 25. One may define the total ratio adding all pair threshold contributions with weights determined by oscillation data [16]; $R_{A}(\omega)=F^{A}\left(\omega ; T_{\nu}\right) / F^{A}(\omega ; 0)\left(F^{A}(\omega ; 0)\right.$ is the rate factor without the Pauli blocking). The theoretically calculated quantity $R_{A}(\omega)$ shown below is insensitive to relevant transition dipole moments and other atomic factors, the atomic dependence being essentially given by $\epsilon_{e g}$ alone. Corresponding experimental values $R_{A}(\omega)$ need input of theoretical calculation of rates without the Pauli blocking, which requires other atomic parameters than $\epsilon_{e g}$.

Calculated theoretical values of the spectral distortion are shown in Fig(1), Fig(2) and Fig(4) for the nuclear monopole contribution and in Fig(3) for the spin current contribution. Effects of non-vanishing CPV phases that appear in the weight factor of pair emission are small, hence for simplicity we assume the vanishing CPV phase in the following analysis. Main results shown in Fig(1) and Fig(4), but neither in Fig(2) nor in Fig(3), are insensitive to which of the neutrino mass hierarchical patterns, the normal or the inverted hierarchy $(\mathrm{NH}, \mathrm{IH})$, is adopted, and results for these two cases are identical.

The Pauli blocking effect becomes the largest in the threshold region of neutrino pair emission of smallest mass $m_{0}$. In Fig(1) Fig(4) we take hypothetical atoms of excitation energy in the range, $0.1 \sim 100 \mathrm{meV}$, and show the Pauli blocking effect given by the rate ratio $R_{A}(\omega)$. The difference between distortions of $1.9 \mathrm{~K}$ and $2.7 \mathrm{~K}$, of a crucial importance to cosmology, may reach 10 per cent level for appropriate combination of $m_{0}$ and $\epsilon_{e g}$, as in Fig(1).

Study of relic neutrino detection becomes more practical after RENP process is discovered for a definite target atom and a range of smallest neutrino mass is identified. Anticipating an approximate $m_{0}$ determination already achieved, we present in Fig(2) the maximal spectral distortion assuming a special relation between $m_{0}$ and the atomic level spacing. The peak structure for $m_{0}$ values of $0,1 \mathrm{meV}$ observed in Fig(2), which shows a large distortion, is due to the second threshold $\omega_{22}$ of the next lightest neutrino pair of mass $\sim 10 \mathrm{meV}$.

At the zero momentum limit of $p=0,1-f_{i} \sim 1 / 2$ with the vanishing chemical potential, and the effect of Pauli blocking becomes the largest. The reason the largest distortion of $3 / 4$ is not realized in RENP unlike the case of inverse process [14] is that at thresholds $\omega_{i j}$ neutrinos cannot carry the zero momentum and only a partial blocking occurs, since the half energy $\sim \epsilon_{e g} / 2$ is shared by two neutrinos.

The absolute value of RENP spectral rates depends linearly on a time varying dynamical factor $\eta_{\omega}(t)$, which is the product of medium polarization and the stored field energy in dimensionless units and may be calculated by solving the master equation of coherence evolution [13. The nuclear monopole contribution [23] gives the largest rate of order 50 events/second $\times \eta_{\omega}(t)$ at its maximum for Xe atomic de-excitation of ${ }^{3} P_{1}(\sim 8.4 \mathrm{eV})$ for a gas target number density $7 \times 10^{19} \mathrm{~cm}^{-3}$ and a target volume $10^{2} \mathrm{~cm}^{3}$. The large rate for a heavy atom $\propto Q_{w}^{2} Z^{8 / 3}$ arises, since the monopole charge is proportional to $Q_{w}=N-0.044 Z$, with $N, Z$ the neutron number and the proton number of nucleus. The rate is further enhanced by a large Coulomb interaction. Dependence on atomic parameters is more complicated, but very roughly the rate scales as $\propto$ the level spacing $\epsilon_{e g} \times$ relevant E1 dipole strength squared. Although the rate near 


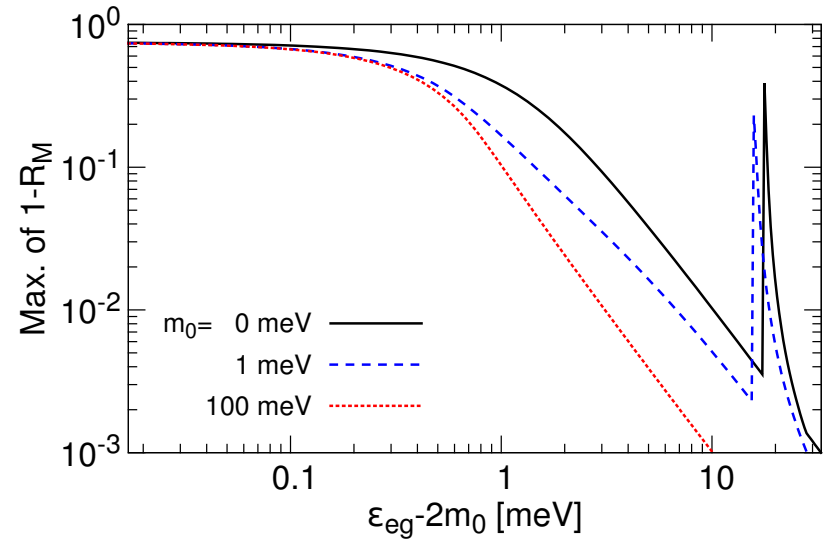

Figure 2: Maxima of the spectral deviation $1-R_{M}(\omega)$ caused by the Pauli blocking plotted against the difference between level splitting and twice of the lightest neutrino mass, $\epsilon_{e g}-2 m_{0}$. We show the cases of NH $m_{0}=0 \mathrm{meV}$ (solid black), $\mathrm{NH} 1 \mathrm{meV}$ (dashed blue) and $\mathrm{NH} 100 \mathrm{meV}$ (dotted red), assuming the zero chemical potential.

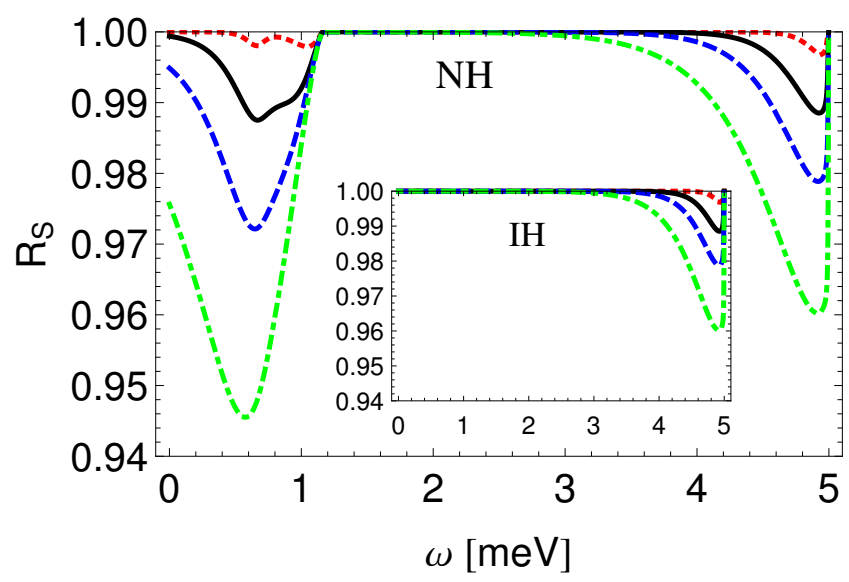

Figure 3: Spectral distortion $R_{S}(\omega)$ caused by the Pauli blocking. $T_{\nu}=1.9 / 2 \mathrm{~K}$ in dotted red, $1.9 \mathrm{~K}$ in solid black, $2.7 \mathrm{~K}$ in dashed blue and $1.9 \times 2 \mathrm{~K}$ in dash-dotted green, all assuming $m_{0}=0.1 \mathrm{meV}, \epsilon_{e g}=10 \mathrm{meV}$ and the zero chemical potential. IH case is shown in the inset for comparison with $\mathrm{NH}$ case. 
the threshold is suppressed, it rapidly increases towards a maximum value at higher photon energies.

Distortions of $10 \%$ level or more seen in Fig(1) and Fig(2) for the nuclear monopole contribution are experimentally encouraging. The distortion of the photon spectrum in the spin current contribution has an interesting second structure as shown in Fig(3) due to the second threshold of the neutrino pair of smallest and the next smallest masses. This second structure is present only in the $\mathrm{NH}$ case, and absent in the $\mathrm{IH}$ case which gives identical results for the right side of structure to the $\mathrm{NH}$ case. Rates are however much smaller than the monopole case [23].

The distorted spectrum for a finite value of the chemical potential has been calculated, as illustrated in Fig(4) [26]. For a choice of small $m_{0}$ effects of the finite chemical potential may be non-negligible.

The prospect of relic neutrino detection is closely tied to a success of neutrino mass spectroscopy using RENP. We shall briefly describe the present status of our project and necessary investigation towards the final goal.

RENP uses the macro-coherence concept [27] which gives the dependence $\propto n^{3} V$ with $n$ the target number density and $V$ the target volume, and the phase matching condition or the momentum conservation among three light particles $\gamma, \nu_{i}, \bar{\nu}_{j}$. The macro-coherence works when more than two light particles are emitted in the final state, giving an important difference from super-radiance that restricts the coherent region to wavelength squared [28. Recently we succeeded in experimentally observing the macro-coherent two-photon emission of a QED process, called Paired Super-Radiance (PSR) [29. This indicates the enhancement mechanism ( $>10^{15}$ in rate) of macro-coherence. A similar, but larger macro-coherence should also work in RENP.

Moreover, the macro-coherent PSR may be used for development of the macro-coherence of RENP, which ultimately leads to formation of static object called soliton-condensate [30]. This is the remnant state of large stored light field coupled to macroscopic medium polarization after the termination of PSR related activity, giving a stationary value of the dynamical factor $\eta_{\omega}$ as large as $10^{-3}$ or even more. In the target state of soliton-condensates two-photon QED backgrounds are exponentially suppressed, thus enhancing the signal to the background ratio.

Initial states of PSR and RENP processes have however different parities, and one needs a switching mechanism between two different parities. One of the ideas for this is the use of external electric field to mix different parity states. Experimental study of targets in solid environments is important to further narrow down a practical way of RENP experiment.

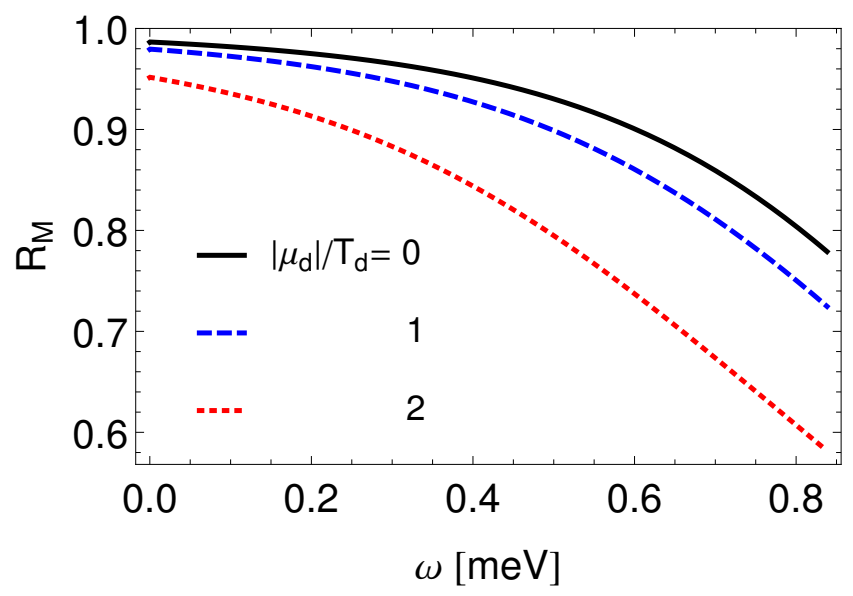

Figure 4: Spectrum distortion $R_{M}(\omega)$ for magnitudes of neutrino degeneracy $\left|\mu_{d}\right| / T_{\nu}=0 \mathrm{meV}$ in solid black, 1 in dashed blue, and 2 in dotted red. The lightest neutrino mass $m_{0}=0 \mathrm{meV} . \epsilon_{e g}=10 T_{\nu} \sim 1.7 \mathrm{meV}$ chosen.

Finally, we mention the directional variation of distorted spectrum. This is caused by the earth motion relative to the $2.7 \mathrm{~K}$ microwave isotropic distribution, giving an effective momentum change of a dipole form in the neutrino distribution function of order given by its velocity $v / c=O\left(10^{-3}\right)$. This effect should help identify relic RENP events near the thresholds.

In summary, neutrino mass spectroscopy using RENP may become a sensitive tool to explore the early cosmic epoch at the decoupling of the electron neutrino. We proposed to use distortion of the photon energy spectrum caused by the Pauli blocking of ambient relic neutrinos. The sensitivity to the background temperature measurement depends on the unknown mass value of lightest neutrino, in relation to the level spacing of excitation. The spectrum distortion may become large, more than significant 10\% level along with this order of temperature distinction of 1.9 and 2.7 $\mathrm{K}$. A small level spacing thus favored may be provided by fine structure splitting of atoms or in molecular rotational transitions. 
We should like to thank K. Inoue and M. Yamaguchi at Tohoku University for valuable discussions. This research was partially supported by Grant-in-Aid for Scientific Research on Innovative Areas "Extreme quantum world opened up by atoms" (21104002) from the Ministry of Education, Culture, Sports, Science, and Technology, and JSPS KAKENHI Grant Number 25400257.

\section{References}

[1] yoshim@okayama-u.ac.jp

† sasao@okayama-u.ac.jp

$\ddagger$ tanaka@phys.sci.osaka-u.ac.jp

[2] For a comprehensive overview of big bang cosmology and nucleo-synthesis, see the textbook of S. Weinberg, Gravitation and Cosmology, John Wiley and Sons, New York (1972).

[3] S. Weinberg, Phys. Rev. 128, 1457 (1962).

[4] P. Langacker, J.P. Leveille, and J. Sheiman, Phys. Rev. D 27, 1228 (1983), and references therein.

N. Cabbibo and L. Maiani, Phys. Lett. 114B, 115 (1982).

[5] L. Stodolsky, Phys. Rev. Lett. 34, 110 (1975).

[6] T.J. Weiler, Phys. Rev. Lett. 49, 234 (1982).

[7] J.M. Irvine and R. Humphreys, J. Phys. G9, 847 (1983).

[8] T. Takahashi and M. Yoshimura, Effect of Relic Neutrino on Neutrino Pair Emission from Metasable Atoms, arXiv:hep-ph/0703019v1 (2007).

[9] R.S. Raghavan, arXiv:hep-ph/0703028 (2007).

[10] A.G. Cocco, G. Mangano, and M. Messina, J. Cosmol. Astropart. Phys. 06 (2007) 015, and arXiv:hep$\mathrm{ph} / 0703075 \mathrm{v} 1$ (2007).

[11] For earlier reviews of relic neutrino detection, see

G. Gelmini, Phys. Scr. T121, 131 (2005):

A. Ringwald, Nucl. Phys. A827, 501c (2009).

[12] On PTOLEMY project, see S. Betts et al, arXiv:1307.4738v2 [astro-ph.IM] (2013).

[13] A. Fukumi et al., Prog. Theor. Exp. Phys. (2012) 04D002, and earlier references cited therein.

[14] In [8] the inverse process of $\gamma+|g\rangle \rightarrow \nu_{i} \bar{\nu}_{j}+|e\rangle$ to RENP was considered, taking into account the Pauli blocking effect. The process is more difficult to experimentally detect than RENP, even if it can be macro-coherently amplified. But the Pauli blocking effect is larger and reaches the largest reduction factor $3 / 4$ in the range $m_{0} \leq$ $1 \mathrm{meV}$ even for larger level spacing $\epsilon_{e g}$.

[15] M Yoshimura, N. Sasao, and S. Uetake, Phys. Rev. D 90, 013022 (2014).

[16] G. L. Fogli, E. Lisi, A. Marrone, D. Montanino, A. Palazzo, and A. M. Rotunno, Phys. Rev. D 86, 013012 (2012).

M. C. Gonzalez-Garcia, M. Maltoni, J. Salvado, T. Schwetz, J. High Energy Phys. 12 (2012) 123.

D. V. Forero, M. Toacutertola, and J. W. F. Valle, Phys. Rev. D 86, 073012 (2012).

[17] One might consider that the chemical potential is defined only in the case of Dirac neutrino in which the particle number is well defined. But weak interaction of Majorana neutrino prior to decoupling distinguishes two different helicity states, which may effectively be regarded as particle and antiparticle as in the Dirac case. Only the gravitational clustering that occurs much later mixes two helicity states, thereby making the chemical potential vanishing. Since we do not need to consider the effect of gravitational clustering, even the Majorana case may be endowed with the chemical potential. 
[18] R.V. Wagoner, W.A. Fowler, and F. Hoyle, Astrophys. J. 148, 3 (1967).

Y. David and H. Reeves, Phil. Trans. R. Soc. Lond. A 296, 415 (1980).

N.C. Rana, Phys. Rev. Lett. 48, 209 (1982).

H. Kang and G. Steigman, Nucl. Phys. B372, 494 (1992).

D.J. Schwarz and M. Stuke, New J. Phys. 15, 033021(2013).

[19] Examples giving a large lepton asymmetry are A. Casas, W.Y. Cheng, and G. Gelmini, Nucl. Phys. B538, 297 (1999):

J. McDonald, Phys. Rev. Lett. 84, 4798 (2000).

[20] A.D. Dolgov, Phys. Rep. 370, 333 (2002).

[21] S. Singh and C.P. Ma, Phys. Rev. D 67, 023506 (2003).

[22] A. Ringwald and Y. Wong, J. Cosmol. Astropart. Phys. 12 (2004) 005.

[23] M. Yoshimura and N. Sasao, Phys. Rev. D 89, 053013 (2014).

[24] D.N. Dinh, S. Petcov, N. Sasao, M. Tanaka, and M. Yoshimura, Phys. Lett. B719, 154 (2012).

[25] We have computed the Dirac neutrino case in a few examples. In the case of Fig(1) there is no appreciable difference between the Majorana and the Dirac cases.

[26] In Fig(4) the case of $m_{0}=0 \mathrm{meV}$ (actually meaning $m_{0} \ll \epsilon_{e g} / 2$ ) is treated, because cases of finite $m_{0}$ values give less distortions for finite chemical potentials. Moreover, one needs a formula of neutrino pair production rates for helicity decomposed neutrino pairs, requiring a more complicated formula than the weighted sum of eq.(1).

[27] M. Yoshimura, N. Sasao, and M. Tanaka, Phys. Rev. A 86, 013812 (2012), and Dynamics of paired superradiance, arXiv:1203.5394 quan-ph] (2012).

[28] R.H. Dicke, Phys. Rev. 93, 99 (1954).

[29] Y. Miyamoto et al, arXiv:1406.2198 v2 [physics.atom-ph] (2014).

[30] M. Yoshimura and N. Sasao, Prog. Theor. Exp. Phys. (2014) $073 B 02$. 